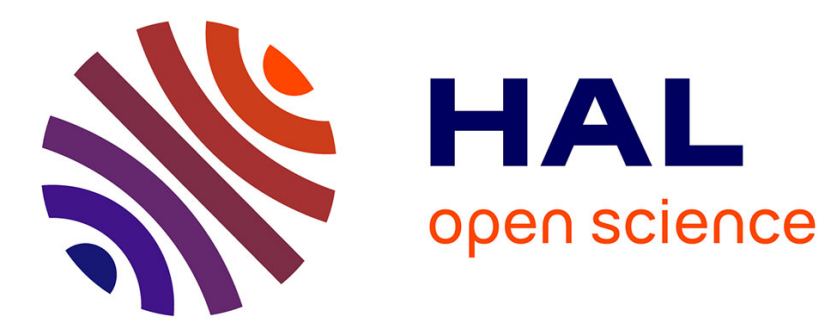

\title{
Beam Bridge Health Monitoring Algorithm Based on Gray Correlation Analysis
}

Jianguo Huang, Lang Sun, Hu Meng

\section{To cite this version:}

Jianguo Huang, Lang Sun, Hu Meng. Beam Bridge Health Monitoring Algorithm Based on Gray Correlation Analysis. 2nd International Conference on Intelligence Science (ICIS), Oct 2017, Shanghai, China. pp.409-416, 10.1007/978-3-319-68121-4_44. hal-01820918

\section{HAL Id: hal-01820918 \\ https://hal.inria.fr/hal-01820918}

Submitted on 22 Jun 2018

HAL is a multi-disciplinary open access archive for the deposit and dissemination of scientific research documents, whether they are published or not. The documents may come from teaching and research institutions in France or abroad, or from public or private research centers.
L'archive ouverte pluridisciplinaire HAL, est destinée au dépôt et à la diffusion de documents scientifiques de niveau recherche, publiés ou non, émanant des établissements d'enseignement et de recherche français ou étrangers, des laboratoires publics ou privés. 


\title{
Beam Bridge Health Monitoring Algorithm Based on Gray Correlation Analysis
}

\author{
Jianguo Huang ${ }^{1 *}$, Lang Sun ${ }^{2}$ and Hu Meng ${ }^{2}$ \\ ${ }^{1}$ Center for Information Technology, Hefei Preschool Education College, Hefei, China \\ ${ }^{2}$ HEFEI City Cloud Data Center Co., Ltd. Hefei, China \\ 71067566 @qq.com
}

\begin{abstract}
Bridge construction investment is huge and the service cycle is long. During the service cycle, the bridge structure not only beared the load effect caused by fatigue damage, but also effected by the natural environment and human damage. Beam bridge is the most kind of bridge built on the highway and had a long-term service in China. The main beam of beam bridge is the main load-bearing component. Real-time evaluation of main beam's health degree will greatly improve the safety of highway transportation. Through the rapid assessment of the main beam of the bridge, it can not only directly reflect whether the deflection of the main beam is beyond the dangerous range and the overall condition of the main beam, but also observe the long-term variation rule of the main beam. The current assessment algorithm only stays in the monitoring of whether the deflection of the main beam is beyond the dangerous range, without a complete assessment combined with massive historical data. Based on the theory of Gray Correlation Analysis and combined with the real time data and historical data of bridge monitoring, we calculate the statistical indicator and morphological indicator of the main beam quickly, and evaluate the comprehensive health indicator of the bridge according to the technical specification in this paper.
\end{abstract}

Keywords: Beam bridge, bridge health assessment, Gray Correlation Analysis

\section{$1 \quad$ Introduce}

In recent years, with the continuous development of computer technology, communication technology, embedded sensors and other technologies, the use of computer systems for automatic health monitoring has become the main method of bridge monitoring. The linear evaluation of bridge main beam is an important indicator which can reflect the safety of the bridge. Through the linear monitoring of the main beam of the bridge, it can not only directly reflect whether the deflection of the main beam is beyond the dangerous range and the working condition of the main beam in the operating state, but also observe the long-term variation rule of the main beam. And the linear evaluation of the main beam of the bridge is of great significance to the bridge bearing capacity detection and the bridge earthquake disaster mitigation. 
The linear evaluation of bridge main beam theory is still in the exploratory stage and without unified evaluation method in our country. The method remains on the level of monitoring whether the original data of the main beam deflection exceeds the risk threshold. There is no standardized assessment of the bridge data.

Gray system theory is a discipline with good theoretical research and application value. Gray Correlation Analysis plays a significant role in theoretical research and application study, which is one of the important parts of Gray system theory. Gray Correlation Analysis is a method of analyzing and determining the degree of correlation between factors or factors on the main behavior of the system through the calculation of gray relational degree. The calculation of Gray Correlation Grade is the basement and an important tool of Gray Correlation Analysis. Therefor the establishment and improvement of the Gray Correlation Model is an important discussion topic of the Gray Correlation Analysis. Gray Correlation Grade theories now catch the attention of the scholars at home and abroad, and become an important branch of future developments and researches of the Gray system study area. Applying the Gray Correlation Grade theory to evaluate the morphological characteristics of the main beam can provide reliable analysis theory support for bridge monitoring.

In this paper, a health assessment algorithm for the main beam of beam bridge based on Gray Correlation Grade is proposed, which can solve the shortcomings that evaluation cannot be synthetically in existing technologies.

\section{$2 \quad$ Health Monitoring Algorithm}

The algorithm mainly includes the following steps :

Step 1: Define the evaluation strategy

Define the range of the outliers and the reference time range of the main beam deflection data.

Step 2: Obtain the original data of deflection

Read original data obtained by the $i$ sensors installed on the main beam in real time, and store them as arrays in memory. The array is defined as rawdata $\{\{i d 0$, time, value $\}$... \{idn, time, value\}\}.

Step 3: Filter the deflection data

Classify the scrolling raw data based on the evaluation strategy, dividing the array rawdata $_{i}$ into the array of reference values refdata $a_{i}$ and the array to be evaluated candata.

The value of the attribute value in the array rawdata $a_{i}$ is filtered based on the range of the outliers of the main beam deflection data, and the arrays in the range of the outliers are discarded and the arrays in the range of the outliers are kept in the array rawdata . $_{\text {. }}$

The value of the attribute time in the array rawdata ${ }_{i}$ is filtered based on the reference time range of the main beam deflection data, and the arrays in the time range of the reference value are divided into the array of reference values refdata, and the array is not within the time range of the reference values candata $_{i}$.

Step 4: Calculate reference values 
Get the reference mean value $a_{i 0}$ and the reference variance value $b_{i 0}$ the reference displacement value $d_{i 0}$ and the reference numerical sequence $L_{0}$ from refdata.

Calculate the base mean $a_{i 0}$ as follows:

$$
\mathrm{a}_{\mathrm{i} 0}=\frac{\text { refdata }_{\mathrm{i} 0}+\operatorname{refdata}_{i 1}+\cdots+\operatorname{refdata}_{i n}}{n}
$$

In formula (1), $i$ is the number of measured points, refdata in is the $n$-th measured value of the $i$-th measure.

Calculate the benchmark variance $b_{i 0}$ as follows:

$$
\mathrm{b}_{\mathrm{i} 0}=\frac{1}{\mathrm{n}} \sum_{\mathrm{j}=1}^{n}\left|\frac{\mathrm{refdata_{ \textrm {ij } } - \mathrm { refdata }} \mathrm{i}_{\mathrm{i}}}{\mathrm{refdata} \mathrm{i}_{\mathrm{i}}}\right|
$$

In formula (2), $i$ is the number of measured points, refdata $a_{i j}$ is the $j$-th measured value of the $i$-th measuring point.

Calculate the reference displacement value $d_{i 0}$ as follows:

$$
\mathrm{d}_{\mathrm{i} 0}=\frac{\mid \text { refdata }_{i 0}-\text { refdata }_{\mathrm{i} 1}|+| \text { refdata }_{i 1}-\text { refdata }_{\mathrm{i} 2}|+\ldots+| \text { refdata }_{i(\mathrm{n}-1)}-\text { refdata }_{\mathrm{in}} \mid}{n}
$$

In formula (3), $i$ is the number of measured points, refdata ${ }_{i n}$ is the $n$-th measured value of the $i$-th measure.

Calculate the displacement monitoring data $L_{0}$. The baseline sequence is calculated as follows:

$$
L_{0}=\left\{a_{00}, a_{10}, \cdots \cdots, a_{i 0}\right\}
$$

In formula (4), $d_{00}$ is the reference mean of the first measurement point, $a_{i 0}$ is the reference mean of the $i$-th measurement point.

Step 5: Evaluate the health characteristics of the main beam indicators

According to the health data of the main beam, calculate the variance variation coefficient, the maximum variance variation coefficient, the maximum displacement change coefficient, the ride coefficient and the curvature change coefficient.

First, calculate the mean change coefficient. Calculate the mean value of the deflection monitoring point along the main beam distribution, and define the mean change coefficient $\Delta a$ as follows:

$$
\Delta \mathrm{a}=\frac{1}{\mathrm{n}} \sum_{\mathrm{i}=1}^{n} \mid \text { candata }_{\mathrm{i}}-\mathrm{a}_{\mathrm{i} 0} \mid
$$

In formula (5), $n$ is point numbers, candata $_{i}$ is the measured value of the $i$-th measuring point, $a_{i 0}$ is the reference mean of the $i$-th measurement point.

Then calculate the variance change coefficient. Calculate the variance change of the overall deflection of the data at the deflection monitoring point along the main beam, and define the variance change coefficient $\Delta b$ as follows: 


$$
\Delta \mathrm{b}=\frac{1}{\mathrm{n}} \sum_{\mathrm{i}=1}^{n}\left|\frac{\mathrm{b}_{\mathrm{i}}-\mathrm{b}_{\mathrm{i} 0}}{\mathrm{~b}_{\mathrm{i} 0}}\right|
$$

In formula (6), $\Delta b$ is the variance change coefficient of the deflection point, $b_{i}$ is the measured variance of the $i$-th measuring point, $b_{i 0}$ is the reference variance.

Then calculate the maximum variance change coefficient. Define the maximum variance change coefficient $\Delta c$ as follows:

$$
\Delta \mathrm{c}=\max \left|\frac{\mathrm{b}_{\mathrm{i}}-\mathrm{b}_{\mathrm{i} 0}}{b_{\mathrm{i} 0}}\right|
$$

In formula (7), $\Delta c$ is the maximum variance change coefficient, $b_{i}$ is the measured variance of the $i$-th measuring point, $b_{i 0}$ is the reference variance.

Then calculate the maximum displacement change coefficient. Define the maximum displacement change coefficient $\Delta \mathrm{d}$ as follows:

$$
\Delta d=\max \left|\frac{d_{i}-d_{i 0}}{d_{i 0}}\right|
$$

In formula (8), $d_{i}$ is the measured displacement of the $i$-th measuring point, $d_{i 0}$ is the reference displacement value.

According to the theory of Gray Correlation Analysis, the ride coefficient $\bar{p}$ is calculated, and the sequence of the $j$-th of the monitoring points is defined as $L_{j}$

$$
L_{j}=\left\{\operatorname{candata}_{j}(1), \operatorname{candata}_{j}(2), \operatorname{candata}_{j}(3), \cdots \cdots, \operatorname{candata}_{j}(\mathrm{i})\right\}
$$

Define the correlation coefficient as $p_{j}$, the formula is as follows:

$$
\mathrm{p}_{\mathrm{j}}=r\left(L_{0}, L_{j}\right)=\frac{1}{i-1} \sum_{k=1}^{i-1}\left(\frac{1}{1+\left|\frac{L_{0}(k+1)-L_{0}(k)}{L_{0}(k+1)}-\frac{L_{j}(k+1)-L_{j}(k)}{L_{j}(k+1)}\right|}\right)
$$

$k=1,2,3 \ldots, i-1$

In formula (10), $L_{0}(k+1)$ is the $k+1$ value of the reference numerical sequence, $L_{0}$ $(k)$ is the $k$-th value of the reference numerical sequence, $L_{j}(k+1)$ is the $k+1$ value of the data sequence to be evaluated, $L_{j}(k)$ is the $k$-th value of the data sequence to be evaluated.

Then normalize the degree of correlation. Take the $p_{j}$ average as a ride coefficient, define smoothness coefficient of $\overline{\mathrm{p}}$ as follows:

$$
\overline{\mathrm{p}}=\frac{\sum_{\mathrm{n}=0}^{j} \mathrm{p}_{n}}{j}
$$


In formula (11), $j$ is the number of sampling points, $\mathrm{p}_{j}$ is the correlation coefficient obtained for the vector corresponding to the $j$-th sample point, $\overline{\mathrm{p}}$ is the ride coefficient.

Then calculate the curvature change coefficient. Through the reference value data to obtain the reference deflection deformation curvature $s_{i}$, define the data to be evaluated deflection deformation curvature $s_{\mathrm{i}}$ as follows:

$$
S_{i}=\mathrm{d}_{\mathrm{i}}^{\prime \prime}=\frac{\mathrm{d}_{i+1}-2 d_{i}+d_{i-1}}{\Delta x^{2}}
$$

In formula (12), $s_{i}$ is the deformation curvature of the $i$-th measuring point, $d_{i+1}, d_{i}$, $d_{i-1}$ are the $\mathrm{i}+1$ measuring point, i point, $\mathrm{i}-1$ measuring point deflection deformation value, $\Delta x$ is the distance between two adjacent measuring points.

The mean value of the difference of the vector is taken as the variation coefficient of curvature, and the variation coefficient of curvature is defined as $\Delta S$, and its formula is as follows:

$$
\Delta \mathrm{s}=\frac{1}{\mathrm{n}} \sum_{i=1}^{n}\left|\frac{S_{i}-S_{i 0}}{S_{i 0}}\right|
$$

The data are scored based on the scoring rules and the weight table. Calculate the health status score $F$, the formula is as follows:

$$
F=S(\Delta \mathrm{a}) w_{a}+S(\Delta \mathrm{b}) w_{\mathrm{b}}+S(\Delta \mathrm{c}) w_{c}+S(\Delta \mathrm{d}) w_{d}+S(\overline{\mathrm{p}}) w_{p}+S(\Delta \mathrm{s}) w_{s}
$$

In formula (14), $F$ is the total score of linear evaluation, full score is 100 points, $S(\Delta a)$ is the mean change coefficient score value, $w_{a}$ is the mean change coefficient weight, $S(\Delta b)$ is the variance change coefficient score value, $w_{b}$ is the variance change coefficient weight, $S(\Delta c)$ is the maximum variance change coefficient $w_{c}$ is the maximum variance change coefficient weight, $S(\Delta \overline{\mathrm{p}})$ is the ride factor coefficient score value, $w_{p}$ is the ride coefficient weight, $S(\Delta S)$ is the curvature change coefficient score value, $w_{s}$ is the curvature change coefficient weight.

\section{Experiment and Result}

The experiment data were randomly selected from July 4 to July 18, 2016, a total of 9 days data of Wohe Bridge in Anhui Province for analysis. The data acquisition frequency is $1 \mathrm{HZ}$. After statistical analysis, calculate the maximum displacement of the monitoring points as shown in Table 3.1, the displacement mean as shown in Table 33.2 . 
Table 3.1. The maximum displacement monitoring table $(\mathrm{mm})$

\begin{tabular}{cccccc} 
Date & $\begin{array}{c}\text { Monitoring } \\
\text { point1 }\end{array}$ & $\begin{array}{c}\text { Monitoring } \\
\text { point2 }\end{array}$ & $\begin{array}{c}\text { Monitoring } \\
\text { point3 }\end{array}$ & $\begin{array}{c}\text { Monitoring } \\
\text { point4 }\end{array}$ & $\begin{array}{c}\text { Monitoring } \\
\text { point5 }\end{array}$ \\
\hline $2016-7-4$ & -1.879 & -1.602 & -0.249 & -20.081 & -16.15 \\
$2016-7-5$ & -2.085 & -1.56 & 0.176 & -9.999 & -16.153 \\
$2016-7-6$ & -1.841 & -1.404 & 0.038 & -17.71 & -15.89 \\
$2016-7-13$ & -2.048 & -1.406 & -0.272 & -21.094 & -16.003 \\
$2016-7-14$ & -82.111 & -58.193 & -58.564 & -60.877 & -60.38 \\
$2016-7-15$ & -2.438 & -1.727 & -0.599 & -20.436 & -16.458 \\
$2016-7-16$ & -2.415 & -1.708 & -0.668 & -9.998 & -16.384 \\
$2016-7-17$ & -82.114 & -58.196 & -58.567 & 2.048 & -60.348 \\
$2016-7-18$ & -2.143 & -1.483 & -0.221 & -8.959 & -15.842
\end{tabular}

Table 3.2. The mean displacement monitoring table ( $\mathrm{mm})$

\begin{tabular}{lccccc} 
Date & $\begin{array}{c}\text { Monitoring } \\
\text { point1 }\end{array}$ & $\begin{array}{c}\text { Monitoring } \\
\text { Point2 }\end{array}$ & $\begin{array}{c}\text { Monitoring } \\
\text { Point3 }\end{array}$ & $\begin{array}{c}\text { Monitoring } \\
\text { Point4 }\end{array}$ & $\begin{array}{c}\text { Monitoring } \\
\text { Point5 }\end{array}$ \\
\hline $2016-7-4$ & -1.81 & -1.54 & -0.14 & -18.43 & -16.01 \\
$2016-7-5$ & -1.80 & -1.36 & 0.02 & -13.83 & -15.76 \\
$2016-7-6$ & -1.82 & -1.39 & 0.02 & -17.25 & -15.85 \\
$2016-7-13$ & -1.98 & -1.39 & -0.24 & -20.04 & -15.96 \\
$2016-7-14$ & -2.01 & -1.38 & -0.13 & -13.97 & -15.86 \\
$2016-7-15$ & -2.25 & -1.63 & -0.47 & -19.40 & -16.26 \\
$2016-7-16$ & -2.21 & -1.65 & -0.50 & -15.05 & -16.22 \\
$2016-7-17$ & -2.11 & -1.46 & -0.24 & -7.12 & -15.78 \\
$2016-7-18$ & -2.13 & -1.47 & -0.21 & -8.63 & -15.82
\end{tabular}

With the data of Table 3.1 and Table 3.2, the mean result were drawn line chart of the main beam under permanent load condition as shown in Fig 3.1. 


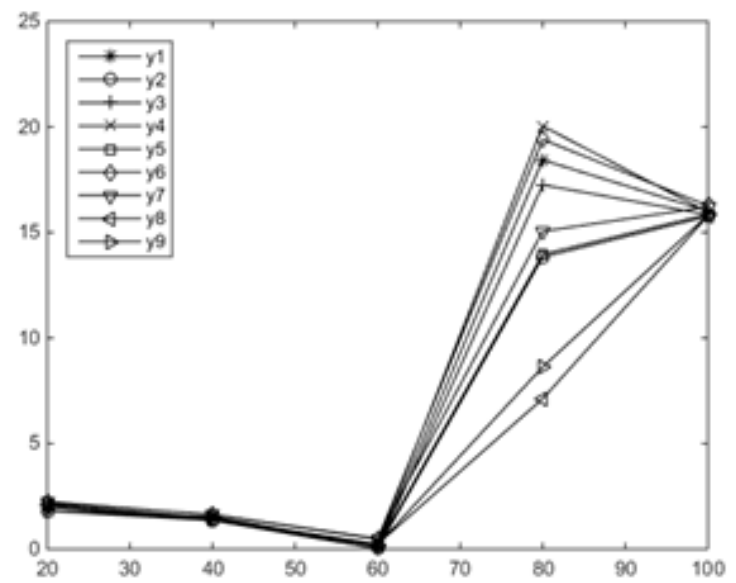

Fig. 3.1. Line chart of main beam

As you can see from Fig 3.1, there was a large fluctuation of displacement at No. 4 monitoring point at $80 \mathrm{~m}$ of the bridge which is under permanent load condition during the monitoring period. So we initially suspected there was something wrong of this bridge. Through the calculation of basic grey relational degree algorithm, the real health data of the bridge was obtained after analysis, the evaluation results are listed in Table 3.4.

Table 3.4. Linear evaluation results table

\begin{tabular}{lllll} 
Ref & $\Delta \mathrm{a}$ & $\Delta \mathrm{b}$ & $\mathrm{ai0}$ & Curv \\
\hline Def1 & -2.11 & 0.70 & -2.11 & 2.00 \\
Def2 & -1.54 & 0.50 & -1.54 & 2.00 \\
Def3 & -0.34 & 0.53 & -0.34 & 2.00 \\
Def4 & -16.45 & 3.65 & -16.45 & 3.00 \\
Def5 & -16.10 & 0.43 & -16.10 & 2.00
\end{tabular}

According to the calculated values, the line chart of the bridge can be evaluated. Referring to 《Rules for inspection and assessment of bearing capacity of Highway Bridges》 (2003) and 《Technical code for highway maintenance》 (JTGH112004) , the technical state of bridge is divided into five categories. The first class is intact and in good state; the second class is in good state; the third class is in poor state; the fourth class is in bad state; the fifth class is in dangerous state. The specific classification criteria are shown in the Table 3.5. 
Table 3.5. Technical condition evaluation

\begin{tabular}{cccccc} 
Grade & I & II & III & IV & V \\
\hline value & $80-100$ & $60-80$ & $40-60$ & $20-40$ & $0-20$
\end{tabular}

From Table 3.5, this bridge's Technical condition evaluation is Grade III, a poor state. It needs a maintenance work.

\section{Conclusion}

The evaluation algorithm proposed in this paper can make the bridge supervisors quick to judge the overall health status of the bridge and the damage status of the main beams compared with the existing technology. This method makes the monitoring data of the bridge have the characteristics of standardization and high efficiency, and improves the robustness and ease of use of the system, and has high engineering application value.

\section{References}

1. Yung-Li Lee, Jwo Pan, Richard B.Hathaway, Mark E. Barkey. Fatigue Testing and Analysis(Theory and Practice). Elsevier, 2005.

2. Stuart L M. Data Analysis for Science and Engineering. John Wily and Sons, 1975

3. Liu Sifeng, Gray System Theory and Application, Science Press, October 2004 (In Chinese)

4. YI Tinghua, Li Hongnan, Gu Ming, Recent Research and Application of GPS Based Technology for Bridge Health Monitoring[J] JSCIENCE CHINA, 2010, 153(10): 25972610(In Chinese)

5. ANG Ying, LI Zhao-xia, Parameter Estimation and Test of Fatigue Stress Probability Distribution Function of Large Span Bridge Based on Monitoring Samples, Modern Traffic Technology, 2008, 5(6): 17-21 (In Chinese)

6. AJ. Morris Foundations of Structural Optimization: A Unified Approach [M]. Wiley lnter Science Publication, John Wiley \& Sons, 1982

7. R. Fox md M. Kapoor. Rate of change of eigenvalues and eigenvectors. AIAA Jouma1, 1968, (6), 24212429.

8. P.W. Mo11er and O. Friberg. Updating Large Finite Element Models in Structural Dynamics AIAA Jouma1, 1998, 36(10), 1861 1868.

9. Zhao J, De WolfJ T, Sensitivity Study for Vibrational Parameters Used in Damage Detection, Jouma1 ofStructura1 Engineering, 1999, 125(4):41()-116.

10. Pmdey A K, Biswas M. Damage Detection in Structures Using Changes in Flexibility, Joumal of Sound and Vibration, 1994, 169(1):3-17. 